\title{
SOI-Based Photonic Crystals
}

\author{
Susumu Noda and Takashi Asano \\ Kyoto University, Kyoto 615-8510, Japan \\ snoda@kuee.kyoto-u.ac.jp
}

\section{Introduction}

Photonic crystals, in which the refractive index changes periodically, provide an exciting new tool for the manipulation of photons and have received keen interests from a variety of fields. In this conference, we would like to review the recent progresses of photonic crystals by focusing on SOI-based two-dimensional (2D) photonic crystals. First of all, some examples of photon manipulation by a combination of line-defect waveguides and point-defect nanocavities are described. Then, the recent remarkable progresses on ultra-high $Q$ nanocavities $(Q=600,000)$ are explained.

\section{Examples of Photon Manipulation with Line and Point Defects in Photonic Crystals}

Figure 1 shows a SEM picture of SOI-based 2D photonic crystal and an example of a manipulation of photons with line and point defects [1], where photons propagating through a line-defect waveguide are trapped by a point-defect cavity and emitted to free space. The phenomenon can be applied to a photonic-nanostructure device as shown in Fig.2(a), where the device consists of in-plane arrays of photonic crystals (in-plane hetero photonic crystals) with a different lattice constant of $1.25 \mathrm{~nm}$ [2]. Multi-channel add/drop operation was indeed achieved as shown in Fig.2(b). It was also shown that the heterointerface between the neighboring photonic crystals plays an important role to achieve a photon manipulation efficiency of $\sim 100 \%$ [3]. A dynamic tuning of resonant wavelength of individual point-defect cavity was also demonstrated by a change of refractive index of the cavity itself [4]. These results clearly indicate that basic optical functions have been successfully

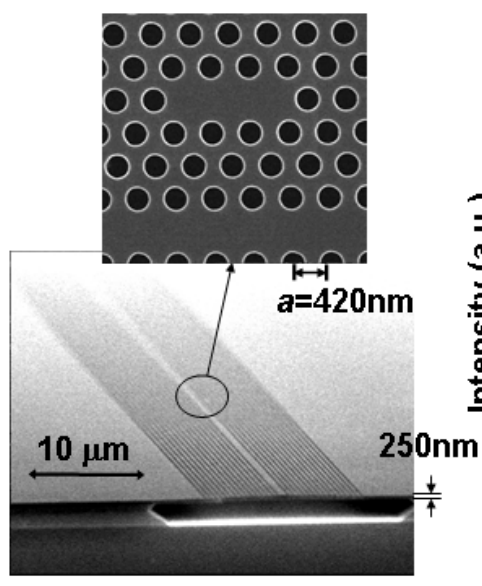

Si on Insulator

(a)

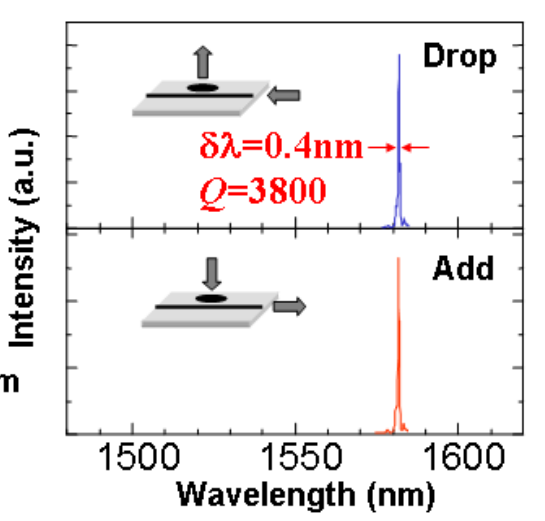

(b)

Fig.1. SOI-based 2D photonic crystal and an example of manipulation of photons with line and point defects: (a) SEM picture and (b) experimental results. demonstrated in SOI-based 2D photonic crystals.

\section{Ultrahigh $Q$ Photonic Nanocavities}

In Fig.1, photons trapped by the point-defect cavity are emitted to the free-space. The cavity $Q$ factor can be estimated to be 3,800 from the spectrum. If we can increase the $Q$ factor drastically, the leakage of photons via the point defect can be reduced and large-scale photonic integration based on line and point defects can be 
constructed. In addition, various other important applications including cavity quantum electrodynamics, quantum communications and computing, photonic buffer memory, and bio and sensing applications will be expected. Thus, there has been much interest in ultrahigh $Q$ nanocavities.

An important design concept to achieve a high- $Q$ nanocavity in $2 \mathrm{D}$ photonic crystals has been recently proposed: the cavity should have a gentle electric field distribution-most ideally like a Gaussian function - to suppress the out-of-slab leakage of photons [5,6]. Based on this concept, unique approach of 'tuning of air-holes' as shown in Fig.3 has been introduced, by which the $Q$ factor up to 45,000 has been obtained [5].

Then, a concept of a photonic doubleheterostructure [7] has been introduced to achieve a further drastic increase of $Q$ factor. Figure 4(a) shows the SEM picture of the fabricated photonic double-heterostructure cavity. Two photonic crystals (PC-I and PC-II) with the different lattice constants of $a_{1}=410 \mathrm{~nm}$ and $a_{1}=420 \mathrm{~nm}$ are joined. As the lattice constants in of PC-I and II vary, the transmission regions of the waveguides in the crystals PC-I and PC-II differ. Therefore, photons with a specific energy can exist only in the waveguide of PC-II and not PC-I due to the mode gap effect. When the waveguide in PC-II is short enough, the frequencies that photons can take in this region become quantized-similar to electrons in a semiconductor quantum structure - and a photonic nanocavity is formed. Because the confinement of photons in the waveguide direction results from the mode-gap effect in the waveguide of PC-I, the evanescent behavior of the electric field which is confined in PC-II, into the waveguide of PC-I, can be

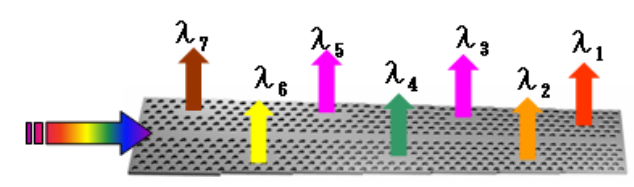

(a)

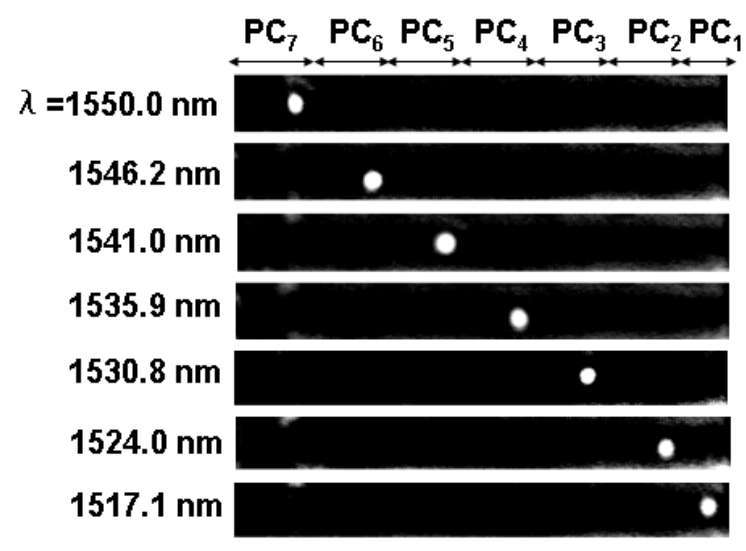

(b)

Fig.2. (a)Photonic-nanostructure device based on in-plane hetero photonic crystals, where in-plane arrays of photonic crystals with different lattice constant $(1.25 \mathrm{~nm})$ are integrated, (b) multi-channel drop (add) operation..

uniformly controlled along the waveguide by changing the lattice-constant difference between the two crystals. The theoretical calculation has proved that the electric field distribution in the double heterostructure nanocavity can be very close to the ideal Gaussian function, which minimizes out-of-slab leakage of photons. The measurement result of the cavity $Q$ is shown in Fig.4(b). The spectrum with an extremely narrow line-width of 
$2.8 \mathrm{pm}$, which was approaching the resolution limit of the measurement system, was obtained. The intrinsic $Q$-factor has been estimated as large as 600,000 , considering the effect of coupling between the cavity and the input waveguide. This was almost of the order of the theoretical value of $10^{6}$.

\section{Summary}

Recent progresses of SOI-based 2D photonic crystals have been reviewed. It has been shown that line and point defects in photonic crystals well manipulate photons, and photonic-nanostructure devices can be indeed realized. Then the progresses of nanocavities with ultrahigh $Q$ factor of 600,000 have been described. The realization of such ultrahigh $Q$ nanocavities should accelerate developments not only in the field of photonics but also in various other fields including quantum communication and computing, bio and accurate environmental monitors.

\section{References}

[1] S. Noda, A.Chutinan, and M. Imada, Nature, $\underline{407}, 608$ (2000).

[2] B.S.Song, S.Noda, and T.Asano, Science, Science, $\underline{300,} 1537$ (2003).

[3] B.S.Song, T.Asano, Y.Akahane, and S.Noda, Physical Review B, 71, 195101 (2005).

[4] T.Asano, W.Kunishi, M.Nakamura, B.S.Song, and S.Noda, Electronics Letters, 41 , 37 (2005).

[5]Y.Akahane, T.Asano, B.S.Song, and S.Noda, Nature, 425, 944 (2003).

[6] T.Asano and S.Noda, Nature, $\underline{429}$, doi:10.1038 (2004).

[7] B.S.Song, S.Noda, T.Asano and Y.Akahane, Nature Materials, $\underline{4}$, 207(2005). 